\title{
ESTIMACIÓN DE PRODUCCIÓN SECUNDARIA Y PRODUCTIVIDAD PARA UNA POBLACION INTERMAREAL DE TROPHON GEVERSIANUS (BAHÍA LAREDO, ESTRECHO DE MAGALLANES).
}

\author{
ESTIMATION OF SECONDARY PRODUCTION AND PRODUCTIVITY FROM AN INTERTIDAL \\ POPULATION OF TROPHON GEVERSIANUS, (LAREDO BAY, STRAITS OF MAGELLAN).
}

Claudia Andrade ${ }^{1,2}$, Américo Montiel $^{2} \&$ Eduardo Quiroga $^{3}$

\section{RESUMEN}

El gastrópodo carnívoro Trophon geversianus (Pallas, 1774) es una de las especies bentónicas explotadas comercialmente en la región de Magallanes, de la cual no existe información relacionada a su productividad biológica. En este trabajo, se estimó la producción secundaria y productividad de una población intermareal de T. geversianus a través del modelo de redes neurales. Los muestreos se realizaron estacionalmente en Bahía Laredo, estrecho de Magallanes entre los meses de agosto 2007 y abril 2008. La longitud de los ejemplares de T. geversianus fluctuó de 12,4 a 55,4 mm (28,4 $\pm 7,4)$. La población presentó una distribución unimodal. Las densidades medias estacionales oscilaron entre 0,23 ind $\mathrm{m}^{-2}$ y 0,97 ind $\mathrm{m}^{-2}$, mientras que la biomasa media varió entre 0,041 g PSLC $\mathrm{m}^{-2}$ y $0,194 \mathrm{~g}$ PSLC $\mathrm{m}^{-2}$. La producción secundaria anual promedio estimada para $T$. geversianus varío entre 0,03 y $0,16 \mathrm{~g}$ PSLC $\mathrm{m}^{-2}$ año-1 y la productividad estimada fue de $0,7 \mathrm{año}^{-1}$. En la razón $\mathrm{P} / \mathrm{B}$ se observó una correlación significativa con el peso corporal de T. geversianus. La productividad estimada para $T$. geversianus es consistente con los valores reportados en la literatura para otras especies de moluscos marinos de las áreas Antártica, Subantártica y Chilenas.

Palabras clave: Gastropoda, Muricidae, razón P/B.

1 Programa de Magíster en Ciencias con Mención Manejo y Conservación de Recursos Naturales en Ambientes Subantárticos, Facultad de Ciencias, Universidad de Magallanes, Chile. claudia.andrade@umag.cl

2 Laboratorio de Hidrobiología, Instituto de la Patagonia, Universidad de Magallanes, Punta Arenas, Chile.

3 Centro de Investigación en Ecosistemas de la Patagonia (CIEP), Coyhaique, Chile. 


\begin{abstract}
Although the carnivorous gastropod Trophon geversianus (Pallas, 1774) is one of the commercially harvested benthic species in the Magellan region, no information is available on its biological productivity. Secondary production and productivity (P/B ratio) from an intertidal population of $T$. geversianus was estimated using the neural networks model. Seasonal sampling was carried out in Bahía Laredo, Estrecho de Magallanes between August 2007 and April 2008. Individual lengths of T. geversianus ranged from 12.4 to $55.4 \mathrm{~mm}(28.4 \pm 7.4)$. The population showed a unimodal size distribution. The seasonal average density ranged from 0.23 ind $\mathrm{m}^{-2}$ to 0.97 ind $\mathrm{m}^{-2}$, while average biomass varied between 0.041 $\mathrm{g}$ AFDW m $\mathrm{m}^{-2}$ and $0.194 \mathrm{~g}$ AFDW m${ }^{-2}$. Mean secondary production was estimated to range between 0.03 and $0.16 \mathrm{~g}_{\text {AFDW m}}{ }^{-2} \mathrm{yr}^{-1}$ and estimated productivity was 0.7 year $^{-1}$. The P/B ratio was significantly correlated with body size of $T$. geversianus. The estimated productivity values of $T$. geversianus are consistent with those reported in the literature for others molluscan species from Antarctic, Subantarctic and Chilean waters.
\end{abstract}

Key words: Gastropoda, Muricidae, P/B ratio.

\section{INTRODUCCIÓN}

El gastrópodo Trophon geversianus (Pallas, 1774), es un murícido endémico de la Provincia Malacológica Magallánica (Linse 1999, Forcelli 2000). Su distribución bioceánica abarca en la costa Atlántica suroccidental desde latitud $36^{\circ} \mathrm{S}$ hasta $56^{\circ}$ $\mathrm{S}$, entre las localidades de Buenos Aires y Tierra del Fuego incluyendo a las islas Malvinas (Falkland Is.) e isla de los Estados. Mientras que en la costa Pacífica suroriental se encuentra desde latitud $42^{\circ}$ $\mathrm{S}$ hasta $56^{\circ} \mathrm{S}$, entre la Isla Grande de Chiloé y el archipiélago del cabo de Hornos (Castellanos \& Landoni 1993, Pastorino 2005, Griffin \& Pastorino 2005). La distribución batimétrica de esta especie se extiende desde el intermareal hasta los $100 \mathrm{~m}$ de profundidad (Ríos \& Gerdes 1997, Gutt et al. 1999, Linse 20001).

Para la costa argentina, Zaixso (1973) y Penchaszadeh (1976) describen aspectos reproductivos en poblaciones naturales no explotadas de T. geversianus, tales como el desove, las características de las ovicápsulas, los estados embrionarios y su nutrición. Santana (1998) describió la época de desove para las costas de la región de Magallanes, como así también el desarrollo intracapsular de la especie bajo condiciones de laboratorio. En tanto,

1 Linse, K. 2000. The shelled Magellanic Mollusca: with special reference to biogeographic relations in the Southern Ocean. Tesis de Doctorado, Fachbereichs Biologie, Universität Hamburg, Alemania.
Santana \& Cañete (2001) describieron los aspectos biométricos de las ovisposturas y determinaron el crecimiento y la mortalidad de individuos juveniles en condiciones de laboratorio.

Como otros murícidos, $T$. geversianus es una especie carnívora. En condiciones de laboratorio es un depredador especialista frente a un determinado ítem presa como Mytilus chilensis (Hupe, 1854) (Andrade 2006², Andrade \& Ríos 2007). Esta preferencia alimenticia por ejemplares de $M$. chilensis coincide con las observaciones realizadas en ambientes naturales por Chávez \& Medina (1998) ${ }^{3}$ y Santana (1998) en el estrecho de Magallanes y por Gordillo \& Amuchástegui (1998) en el canal Beagle. En cuanto a los aspectos biológico-pesqueros de poblaciones de $T$. geversianus, Guzmán et al. (1997) ${ }^{4}$ describieron la talla crítica, mortalidad y la relación talla-peso.

2 Andrade, C. 2006. Relaciones tróficas en invertebrados marinos del estrecho de Magallanes: Estudio experimental de la preferencia alimentaria del gastrópodo Trophon geversianus (Pallas, 1774). Tesis de grado en Licenciatura en Ciencias Biológicas, Facultad de Ciencias, Universidad de Magallanes, Chile.

3 Chávez, J. \& M. Medina 1998. Estudio demográfico del caracol Trophon geversianus (Pallas, 1774) y estimaciones de abundancia para especies de importancia comercial en la zona norte de bahía Gente Grande, provincia de Tierra del Fuego. Informe Servicio País -Chile.

4 Guzmán, L., M. Rojas, S. Oyarzún \& G. Jerez. 1997. Estudio biológico pesquero del caracol: Trophon (Trophon spp.), Piquilhue (Adelomelon ancilla) y Picuyo (Odontocymbiola magellanica). Informe Final FIP 94-28. 
Chávez \& Medina (1998) 5 estudiaron los patrones de distribución, abundancias y la estructura de tamaños de la población de Trophon en bahía Gente Grande.

En la región de Magallanes, T. geversianus se comenzó a explotar comercialmente a partir de 1990 (SERNAPESCA 2008) ${ }^{6}$. Recientemente, González et al. (2007) 7 han descrito el estado de explotación del recurso Trophon a partir de parámetros de crecimiento, mortalidad y reclutamiento. $\mathrm{Al}$ respecto, se ha descrito que $T$. geversianus presenta un reducido potencial reproductivo con crecimiento lento y longevo alcanzando una talla comercial $(60$ $\mathrm{mm}$ ) entre los 3 a 4 años de edad. Además, como consecuencia de esta actividad extractiva se ha observado una drástica disminución en las abundancias poblacionales de la especie (e.g. bahía Gente Grande, González et al. 2007)7.

Los aspectos biológicos más desconocidos de T. geversianus son aquéllos relacionados con la productividad biológica y el rol que desempeña la especie en las diferentes comunidades que integra. De las cinco especies de murícidos explotados en Chile, sólo se cuenta con estudios de productividad para Concholepas concholepas (Bruguière, 1789). En este contexto, Stotz \& Pérez (1992), destacan la necesidad de conocer la producción secundaria y productividad de un recurso para determinar variables como la extracción máxima y la capacidad de carga de un área determinada. De hecho, la producción secundaria es considerada como una de las variables de mayor importancia para evaluar la sustentabilidad de explotación de una población. Esto debido a que incorpora variables tales como la abundancia y la biomasa, las cuales resultan de la energía utilizada para el crecimiento y reproducción de la población (Prieto et al. 2004, Acosta \& Prieto 2008). En consecuencia, el objetivo de este trabajo fue estimar la producción secundaria $(\mathrm{P}) \mathrm{y}$

5 Chávez, J. \& M. Medina 1998. Estudio demográfico del caracol Trophon geversianus (Pallas, 1774) y estimaciones de abundancia para especies de importancia comercial en la zona norte de Bahía Gente Grande, Provincia de Tierra del Fuego. Informe Servicio País -Chile.

6 SERNAPESCA 2008. Anuario estadístico de pesca. Servicio Nacional de Pesca. Ministerio de Economía Fomento y Reconstrucción, Chile.

7 González, J., E. Daza, L. Guzmán, C. Vargas, C. Cortes \& H. Miranda 2007. Diagnóstico para la administración y conservación del recurso caracol Trophon en Bahía Gente Grande, XII Región. Informe Final FIP 2004-47. productividad (razón $\mathrm{P} / \mathrm{B}$ ) de una población de $T$. geversianus en una zona intermareal del estrecho de Magallanes. Adicionalmente, se entrega información sobre aspectos poblacionales de la especie tales como estructura de tallas, densidad y biomasa.

\section{MATERIAL Y MÉTODOS}

\section{Descripción del área de estudio}

El área de estudio se encuentra ubicada en bahía Laredo $\left(52^{\circ} 57^{\prime} \mathrm{S}-70^{\circ} 51^{\prime} \mathrm{W}\right)$, localizada en la costa occidental del sector oriental del estrecho de Magallanes, distante a $25 \mathrm{~km}$ al norte de la ciudad de Punta Arenas (Fig. 1). En bahía Laredo se delimitó un área de estudio en el intermareal, la cual abarca $10 \mathrm{~km}^{2}$ desde cabo Porpesse al oeste. En este sector, el intermareal está constituido geomorfológicamente por una terraza de bloques y cantos, los cuales están insertos en una matriz de sedimentos dominando las gravas arenosas y arenas gravosas. Ambos componentes sedimentológicos fueron originados por el arrastre glaciar (Prieto 1988). La temperatura superficial promedio del agua oscila entre $5,8^{\circ} \mathrm{C}$ en invierno y $10,5^{\circ} \mathrm{C}$ en verano, mientras que la salinidad superficial promedio fluctúa entre 34,3 psu en verano y 36,0 psu en invierno (Andrade, en prep.).

\section{Recolección y análisis de las muestras}

Los muestreos fueron realizados estacionalmente durante la bajamar entre los meses de agosto 2007 y abril 2008. Para realizar el muestreo el área de estudio se subdividió en cuatro subáreas de $2,5 \mathrm{~km}^{2}$ cada una. En cada subárea se estableció digitalmente una grilla de 100 puntos de intersección con un GPS Garmin Etrex y mediante el programa ArcView GIS 3.2, lo cual proporcionó cuadrantes de muestreo de aproximadamente $25 \mathrm{~m}^{2}$ (5 x $5 \mathrm{~m}$; Fig. 1). En cada subárea se seleccionaron cuatro cuadrantes al azar (georeferenciados mediante GPS, Datum WGS 84), en donde se recolectaron todos los especímenes de T. geversianus posibles $\left(\mathrm{N}_{\text {cuadrantes }}=16 ; 4\right.$ por estación del año), los que fueron congelados a $-20^{\circ} \mathrm{C}$. Para conocer la estructura de tamaños de la población de T. geversianus, todos los especímenes fueron medidos (longitud total, LT) con un pie de metro digital $(0,01$ $\mathrm{mm}$ de precisión). Posteriormente, se elaboraron 


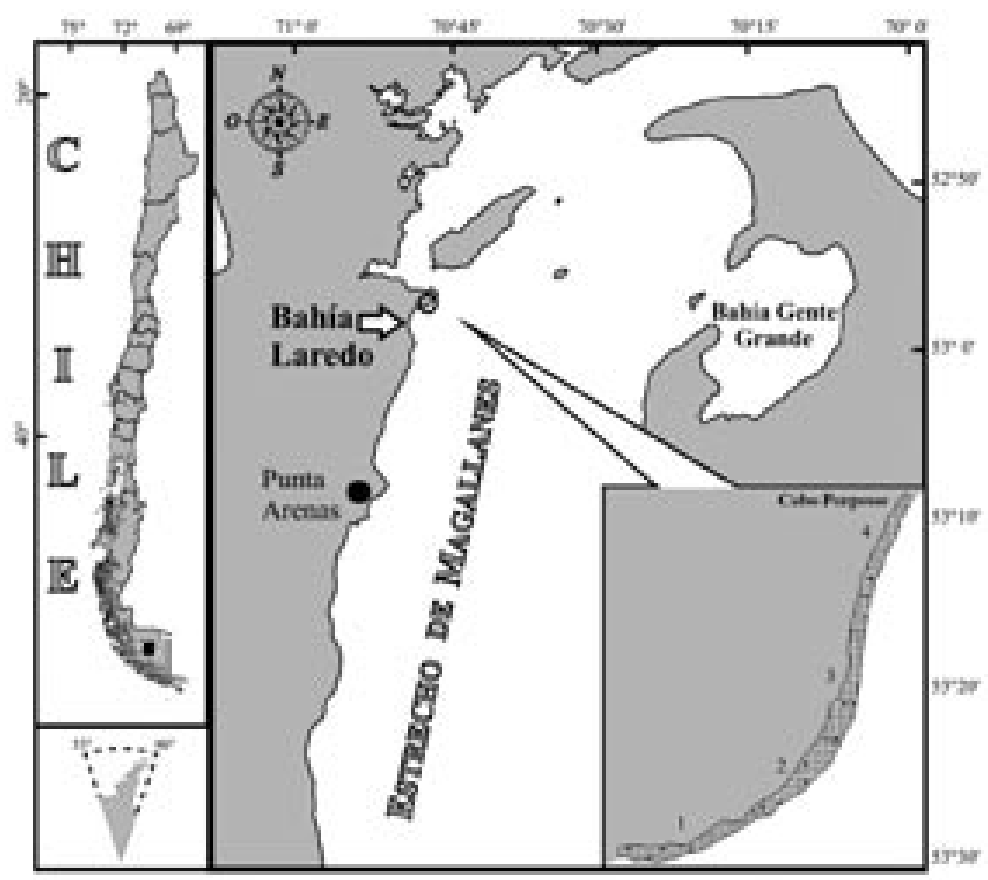

Fig. 1. Ubicación del área de estudio en el intermareal de bahía Laredo, estrecho de Magallanes (Chile).

histogramas de frecuencia de tallas utilizando el programa StatView v5.0.1. Para calcular la biomasa en gramos de peso seco libre de cenizas por metro cuadrado (g PSLC $\mathrm{m}^{-2}$ ), cada espécimen fue pesado libre de su concha en una balanza digital de 0,001 g de precisión y llevado a una estufa a $80^{\circ} \mathrm{C}$ por $12 \mathrm{~h}$ (Asmus 1987). Seguidamente, cada espécimen fue calcinado en una mufla por $24 \mathrm{~h}$ a $550{ }^{\circ} \mathrm{C}$ (Asmus \& Asmus 1990). Todos los análisis fueron realizados en el Laboratorio de Hidrobiología del Instituto de la Patagonia, Universidad de Magallanes.

Para el cálculo de la densidad poblacional (ind $\mathrm{m}^{-2}$ ) se contabilizó el total de ejemplares encontrados en cada cuadrante, este valor fue estandarizado a metro cuadrado y se calculó un promedio para cada estación del año. Los gráficos box plots, fueron realizados utilizando el programa Statistica v7.0.

Estimación de la producción

secundaria y productividad

Se estimó la productividad (razón $\mathrm{P} / \mathrm{B}$ año $\mathrm{o}^{-1}$ ) de T. geversianus mediante un modelo empírico basado en redes artificiales neuronales. Este método se basa en un sofisticado modelo de regresiones múltiples para la estimación anual de producción secundaria y productividad de poblaciones de invertebrados bentónicos (ver Brey et al. 1996, Brey 2001).

El modelo está constituido por los siguientes componentes:

$\log \mathrm{P} / \mathrm{B}=8,256-2,226 \log \mathbf{W}-2432,055$

$(1 / \mathbf{T}+273)+0,239(1 / \mathbf{D})+0,241$ (D E-Subt $)+$ 0,203 (DL In-Epi) $+0,242$ (DL ME) - 0,287 (DT M) - 0,203 (DT P) - 0,128 (DT C) - 0,457(DT E) - 0,116(DH L)

donde, $\mathbf{W}$ es el promedio del peso corporal (kJ), T es la temperatura superficial del agua de mar $\left({ }^{\circ} \mathrm{C}\right)$, D es la profundidad (m). Las categorías, (D E-Subt) especies submareales, (DL In-Epi) infauna-epifauna, (DL ME) móviles epifauna, (DT M) Mollusca, (DT P) Polychaeta, (DT C) Crustacea, (DT E) Echinodermata y (DH L) Lago, se incluyen como variables "dummy" a las que se le asigna el valor 0 si la respuesta es (no) y 1 si la respuesta es (si).

Para la estimación de $\mathrm{P} / \mathrm{B}$, el peso individual y la biomasa de los especímenes de T. geversianus fueron transformadas a kilo Joule utilizando factores de conversión estándar publicados por Brey (2001). La producción secundaria anual (P), se estimó a partir de la multiplicación de la biomasa por la razón P/B. La unidad de expresión de $\mathrm{P}$ anual fue en g PSLC $\mathrm{m}^{-2}$ año-1 $^{-1}$. La relación alométrica entre 
la productividad y el peso corporal (kJ) se realizó usando una regresión lineal simple por medio del programa StatView v5.0.1. Para construir el modelo, se utilizó la razón $\mathrm{P} / \mathrm{B}$ estimada y el peso corporal promedio, en cada cuadrante de muestreo para cada estación del año $(\mathrm{N}=16)$.

\section{RESULTADOS}

\section{Estructura de tallas}

Se recolectó un total de 209 ejemplares de T. geversianus, cuya longitud total fluctuó entre 12,4 y $55,4 \mathrm{~mm}$, con un promedio de $28,4 \pm 7,4$. La población presentó una estructura de tallas unimodal compuesta principalmente por ejemplares juveniles, predominando aquellos que midieron entre 10 y 40 mm de LT ( $\mathrm{N}=199 ;$; 95\%) y se observó baja presencia de individuos de tallas mayores a $40 \mathrm{~mm}(\mathrm{~N}=10$; 5\%). La distribución de tallas de T. geversianus en invierno del 2007 varió entre 12,6 y 46,7 mm, con

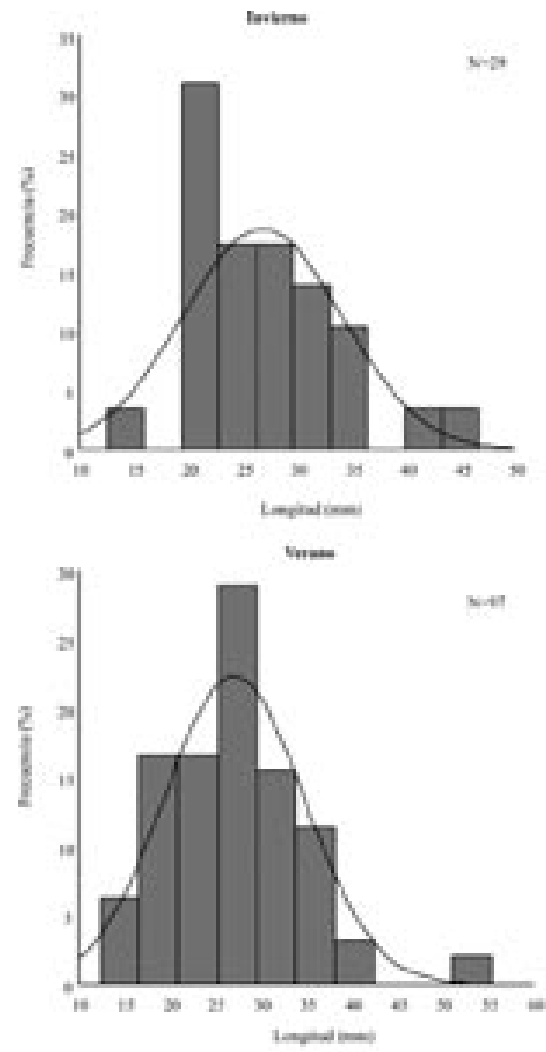

el 93,1\% de la población concentrada entre los 10 y $40 \mathrm{~mm}$. En la primavera de 2007, la población estuvo representada en un $47,8 \%$ por tallas comprendidas entre 25 y $30 \mathrm{~mm}$ y no se observaron tallas mayores a $40 \mathrm{~mm}$. En verano de 2008, se observó un aumento en las frecuencias de tallas, con una mayor incorporación a la población de juveniles entre 10 y $40 \mathrm{~mm}$ (95\%) y con un 3,3\% de ejemplares mayores a $50 \mathrm{~mm}$ siendo la talla máxima 55,3 mm. En otoño de 2008 se observó una tendencia similar a aquella de verano, con sólo un 3,3\% de presencia de ejemplares mayores a 50 $\mathrm{mm}$ (talla máxima $=55,4 \mathrm{~mm}$ ) y con un $95,0 \%$ de representación de ejemplares entre 20 y $40 \mathrm{~mm}$. (Fig. 2).

\section{Densidad poblacional media y biomasa}

La densidad poblacional media total de $T$. geversianus obtenida en este estudio fue 0,52 ind $\mathrm{m}^{-2}$, alcanzando un máximo de 0,97 ind $\mathrm{m}^{-2}$ en

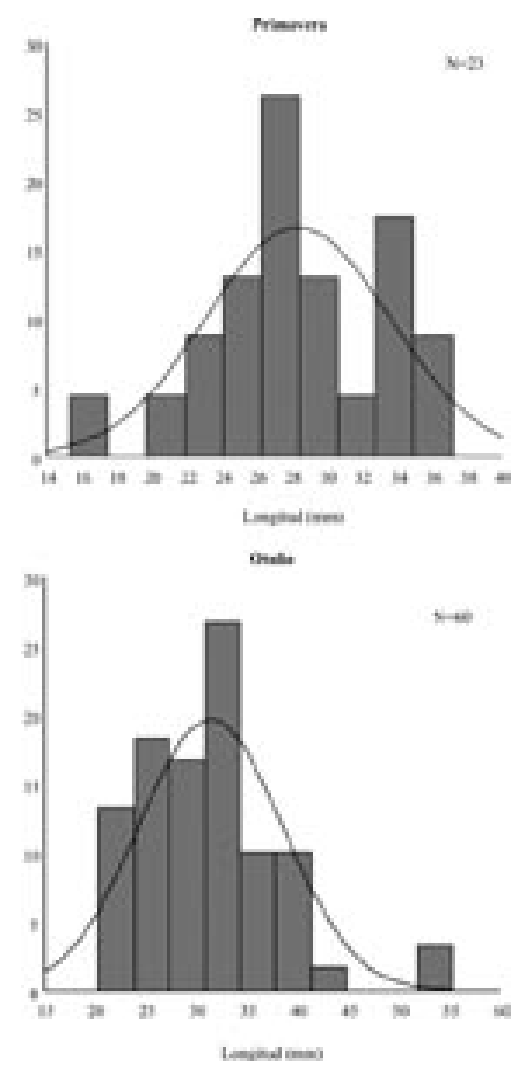

Fig. 2. Frecuencia porcentual (\%) estacional de tamaños (LT) encontradas para Trophon geversianus en el intermareal de bahía Laredo, estrecho de Magallanes (Chile). 

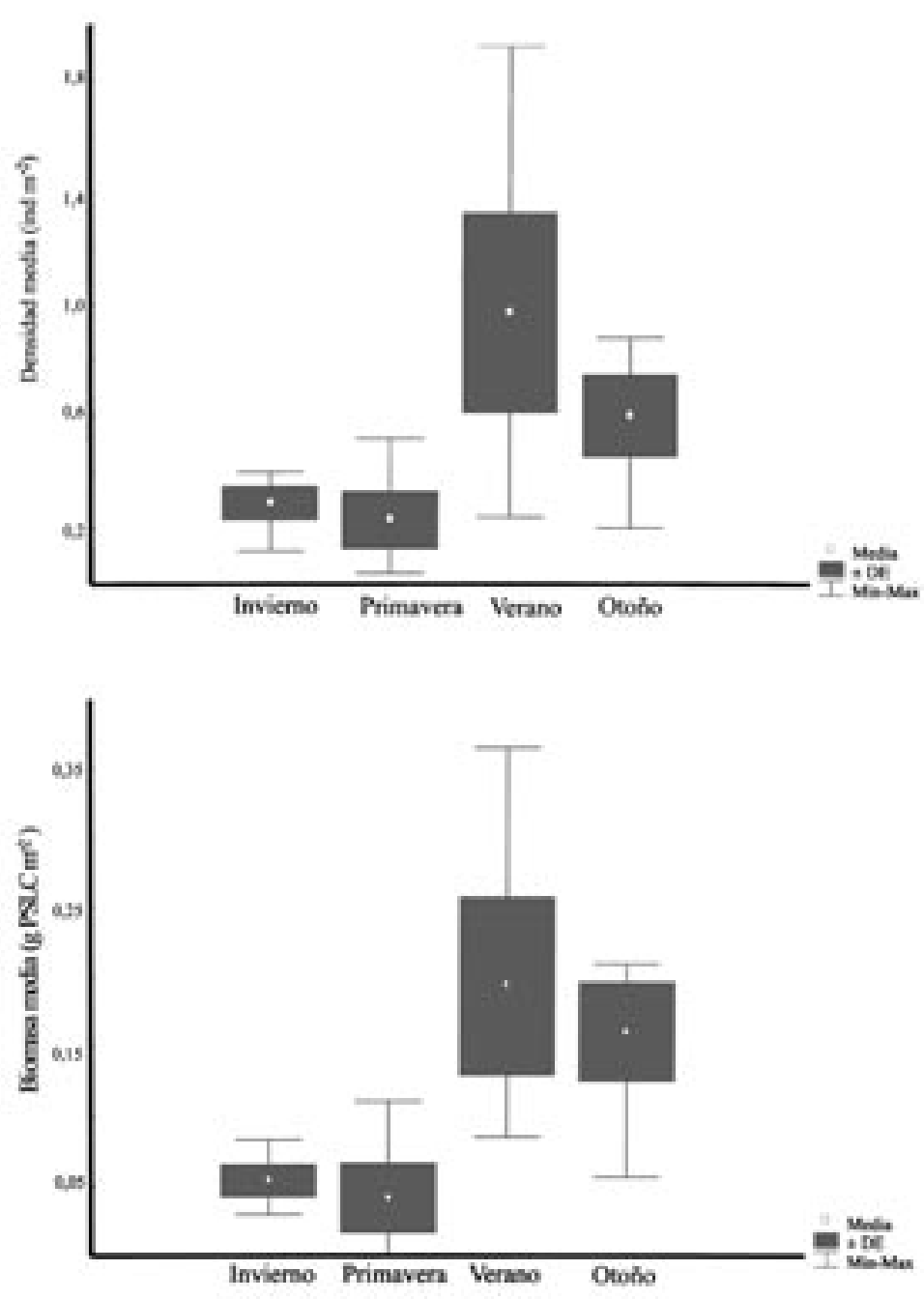

Fig. 3. Densidad y biomasa media $( \pm \mathrm{SE})$ de Trophon geversianus en el intermareal de bahía Laredo, estrecho de Magallanes (Chile).

verano y un mínimo de 0,23 ind $\mathrm{m}^{-2}$ en primavera (Fig. 3a). La biomasa media total fue de 0,112 g PSLC $\mathrm{m}^{-2}\left(0,106 \mathrm{~g} \mathrm{C}\right.$ org $\left.\mathrm{m}^{-2}\right)$, con un mínimo de $0,041 \mathrm{~g}$ PSLC $\mathrm{m}^{-2}$ en primavera y un máximo de $0,194 \mathrm{~g}$ PSLC $\mathrm{m}^{-2}$ en verano (Fig. 3b). Asimismo, la biomasa media total expresada en kilo Joule fue $2,59 \mathrm{~kJ} \mathrm{~m}^{-2}$.

\section{Producción secundaria y productividad}

La producción secundaria anual promedio estimada para T. geversianus osciló entre 0,04 y $0,19 \mathrm{~g}$ PSLC $\mathrm{m}^{-2} \mathrm{año}^{-1}$, y la productividad (P/B) fue de 0,7 año-1 $^{-1}$ Tabla 1 ). Se observó una tendencia significativa $(P=0,0008)$ en la relación razón $P / B$ y peso corporal de $T$. geversianus (Fig. 4), la que indica que a menor peso corporal, mayor es la razón P/B estimada por el modelo.

\section{DISCUSIÓN}

En relación con la estructura poblacional en el área de estudio, la composición por tallas de $T$. geversianus revela la presencia de ejemplares juveniles $(<40 \mathrm{~mm} \mathrm{LT})$. Estos resultados difieren con aquellos informado por González et al. (2007) ${ }^{5}$, quienes no reportaron presencia de juveniles en el banco explotado del sublitoral de bahía Gente Gran- 


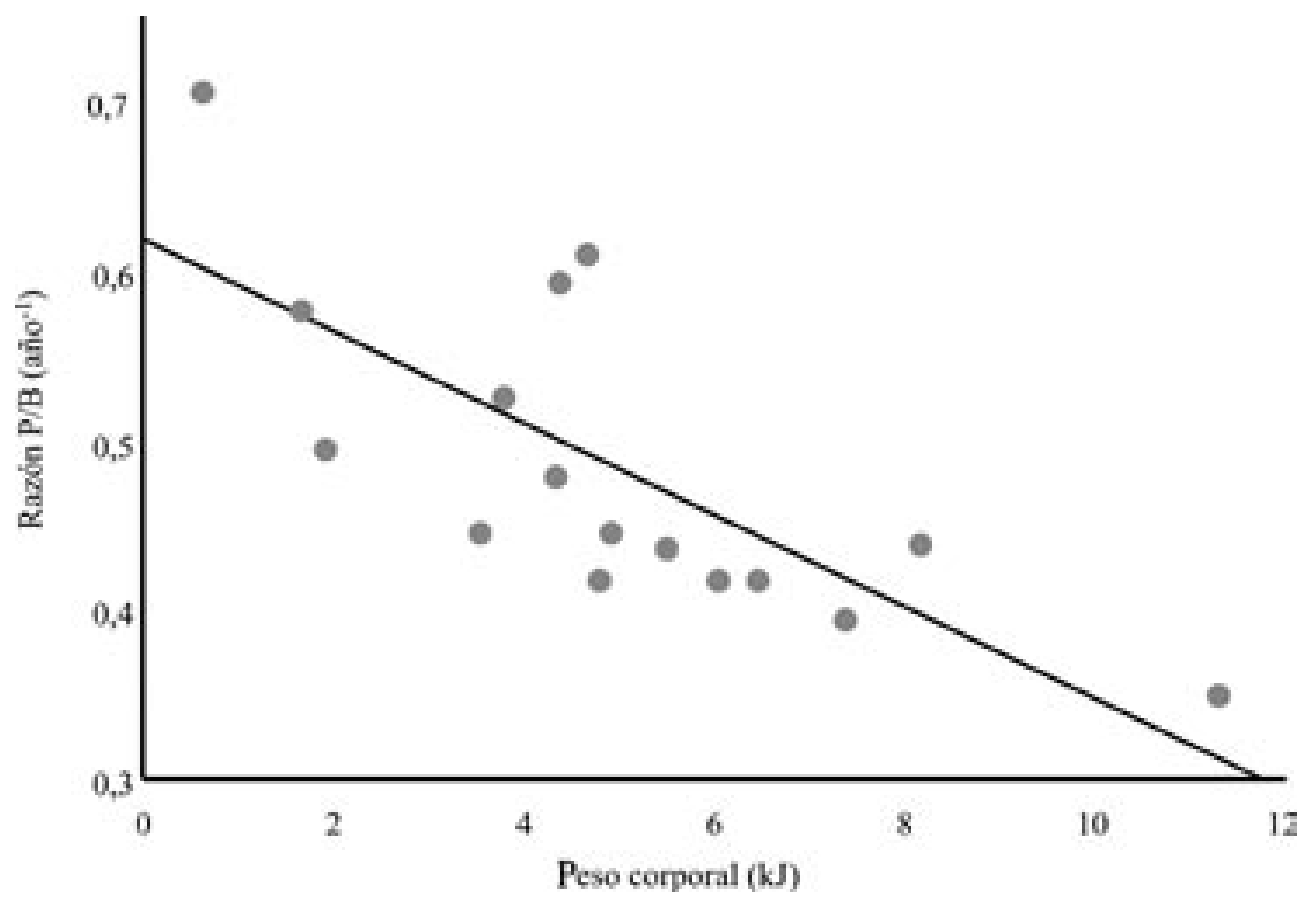

Fig. 4. Relación entre la productividad $(\mathrm{P} / \mathrm{B}$ año-1) y el peso corporal $(\mathrm{kJ})$ para Trophon geversianus en el intermareal de bahía Laredo, estrecho de Magallanes (Chile). Parámetros de la regresión alométrica $\mathrm{N}=16 ; \mathrm{r}^{2}=0,564$; intercepto $=0,618$; pendiente $=-0,027$ y $\mathrm{p}=0,0008$.

de, condición que estaría asociada con el deterioro del recurso por sobreexplotación (e.g. Castilla \& Fernández 1998, Leiva \& Castilla 2002, Rodríguez et al. 2002).

Se ha señalado que la ausencia de estructuras de tallas mayores, en un ambiente intermareal no explotado, estaría asociada con la preferencia de los adultos por habitar los ambientes sublitorales (Prince et al. 1988, Gluyas-Millán et al. 2000). En este sentido, se ha mencionado que $T$. geversianus tiene una distribución espacial diferencial en sus poblaciones, la cual estaría caracterizada por la utilización de un determinado tipo de hábitat por los individuos pequeños, distinto de aquel que utilizan los individuos más grandes (Guzmán et al. 19976). Esto estaría relacionado con el proceso de ovoposición, el cual ocurre en el sublitoral sobre estructuras inertes o sobre sus propias conchas (Guzmán et al. 19976). Luego procede la eclosión de los juveniles, condición por la que podría esperarse un proceso de emigración de individuos juveniles de T. geversianus hacia el intermareal. La condición alternativa sería que las hembras ovígeras se desplazaran al intermareal para depositar las cápsulas en la cara inferior de los bolones para disminuir los riesgos de mortalidad por predación. Este comportamiento se ha sugerido para diferentes gastrópodos marinos (e.g. Vermeij 1972, Schmitt 1981).

La densidad poblacional media de T. geversianus determinada en base a los ejemplares del intermareal de bahía Laredo $\left(0,52\right.$ ind $\left.\mathrm{m}^{-2}\right)$ es baja si se compara con los valores reportados en el sublitoral de bahía Gente Grande (8,40 ind $\mathrm{m}^{-2}$, Chávez \& Medina 19973). Nosotros sugerimos que estas diferencias estarían relacionadas con el comportamiento reproductivo de la especie, debido a que durante las épocas reproductivas de esta especie forma agrupaciones que alcanzan altas densidades durante el verano (González et al. 20075). Sin embargo, estas agrupaciones no fueron observadas en el intermareal de bahía Laredo, debido a que lo juveniles no tendrían este comportamiento.

Se observó que la densidad y biomasa media de T. geversianus decrecen en primavera, estación del año que coincide con los meses en que la especie se encuentra en periodo de desove de las ovicápsulas (Santana 1998), aún cuando en este estudio, no se observaron claros eventos de desove, debido 
probablemente a la gran cantidad de ejemplares en estadios iniciales de madurez (talla madurez sexual en machos $=38,5 \mathrm{~mm}$ y en hembras $=60,2 \mathrm{~mm}$, Guzmán et al. 19976). Posteriormente, la población se recupera en verano, estación en que se observó la mayor biomasa y densidad media, condición derivada del periodo de madurez gonadal de la especie e incorporación de reclutas a la población (Santana 1998).

En general, los métodos más utilizados para estimar la producción secundaria tales como el método de cohorte se basan en información generada a partir de series de tiempo que involucran la medición de variables tales como abundancias poblacionales y tamaño corporal (Crisp 1984, Dolbeth et al. 2005). Sin embargo, este tipo de aproximaciones requieren de tiempo y consumen una gran cantidad de recursos en los programas de muestreo (Gray \& Elliot 2009). En este contexto, los modelos empíricos se han revelado como una herramienta útil que permite incorporar en forma eficiente la interacción de las variables ambientales con la producción secundaria bentónica, y con las variables bióticas como formas de alimentación y movilidad (Tumbiolo \& Downing 1994, Brey 2001, Dolbeth et al. 2005). Para T. geversianus se obtuvo una producción secundaria anual promedio que osciló entre 0,04 y 0,19 g PSLC m ${ }^{-2}$ año-1 y una productividad de $0,7 \mathrm{año}^{-1}$. Estos valores se encuentran en el rango de aquellos reportados por Brey \& Clarke (1993) para distintos taxa, utilizando diversos métodos de análisis (Tabla 1). De hecho, los valores registrados en este estudio coinciden con aquéllos reportados por Brey \& Gerdes (1999) para la región de Magallanes a través del método de redes neurales $\left(0,7 \mathrm{año}^{-1}\right)$. En general, la productividad es un parámetro que depende fuertemente de los variables ambientales como temperatura y profundidad (Sprung 1993, Brey \& Clarke 1993, Cusson \& Bourget 2005). Se ha observado que la productividad estimada para moluscos marinos en las áreas Antártica, sub-Antártica y Chilena, varía entre 0,20 y 1,71 año-1, siendo menor en bivalvos y mayor en gastrópodos (Tabla 1). Esto se relaciona a las diferencias en los patrones de historia de vida de los organismos. Por ejemplo $T$. geversianus es una especie carnívora y móvil, la cual presumiblemente necesita de altos requerimientos metabólicos para su mantención y crecimiento, en marcado contraste con los bivalvos, que siendo organismos filtradores y sésiles, pueden mantener biomasas altas. Es importante destacar que la productividad fue obtenida de una población dominada por juveniles, por lo cual nuestros valores serían más altos que los de una población dominada por adultos.

La razón $\mathrm{P} / \mathrm{B}$ obtenida es concordante para T. geversianus ya que presenta una historia de vida holobentónica, con desarrollo embrionario prolongado en la naturaleza (120 a 150 días, Santana 1998), y un crecimiento de sólo $19 \mathrm{~mm}$ para el primer año, bajo condiciones de laboratorio (Santana \& Cañete 2001). Específicamente, la productividad de T. geversianus $\left(0,7 \mathrm{año}^{-1}\right)$ se aproxima a la de un referente de la misma familia, Concholepas concholepas (0,9 año-1, Stotz \& Pérez 1992) y a las otras especies subantárticas. De lo anterior, se desprende que $T$. geversianus al ser una especie de larga vida, con periodo de crecimiento extendido (Guzmán et al. 19976), con baja productividad, tamaño máximo corporal promedio relativamente menor y selectividad por un único ítem presa, presentaría una condición muy vulnerable si lo contrastamos con C. concholepas, especie que ha estado sometida a una explotación intensiva en Chile por más de 20 años, pero cuya historia de vida pareciera permitir mayores condiciones favorables para su explotación (i.e. crecimiento más rápido, mayor productividad, desarrollo larvario y generalista de presas).

En consecuencia, para los estudios sobre el manejo sustentable de recursos bentónicos marinos sería razonable plantear nuevas estrategias que incluyan conocer ciertos índices ecológicos como producción secundaria y productividad (Stotz \& Pérez 1992, Urban \& Campos 1994). Además, sería necesario estudiar no sólo la especie explotada en cuestión, si no también considerar los impactos indirectos derivados de la alteración del recurso trófico. En el caso de T. geversianus, sería necesario estudiar a su presas $M$. chilensis, recurso que también es explotado en la región. Si no son consideradas estas variables ecológicas pueden resultar un deterioro del recurso y una baja sustentabilidad en el tiempo, lo cual puede ser la causa de la disminución en las abundancias poblacionales de T. geversianus ocurrida en bahía Gente Grande, Tierra del Fuego.

Con respecto a la información disponible para el gastrópodo T. geversianus, aproximadamente el $50 \%$ de los antecedentes biológicos y ecológicos 


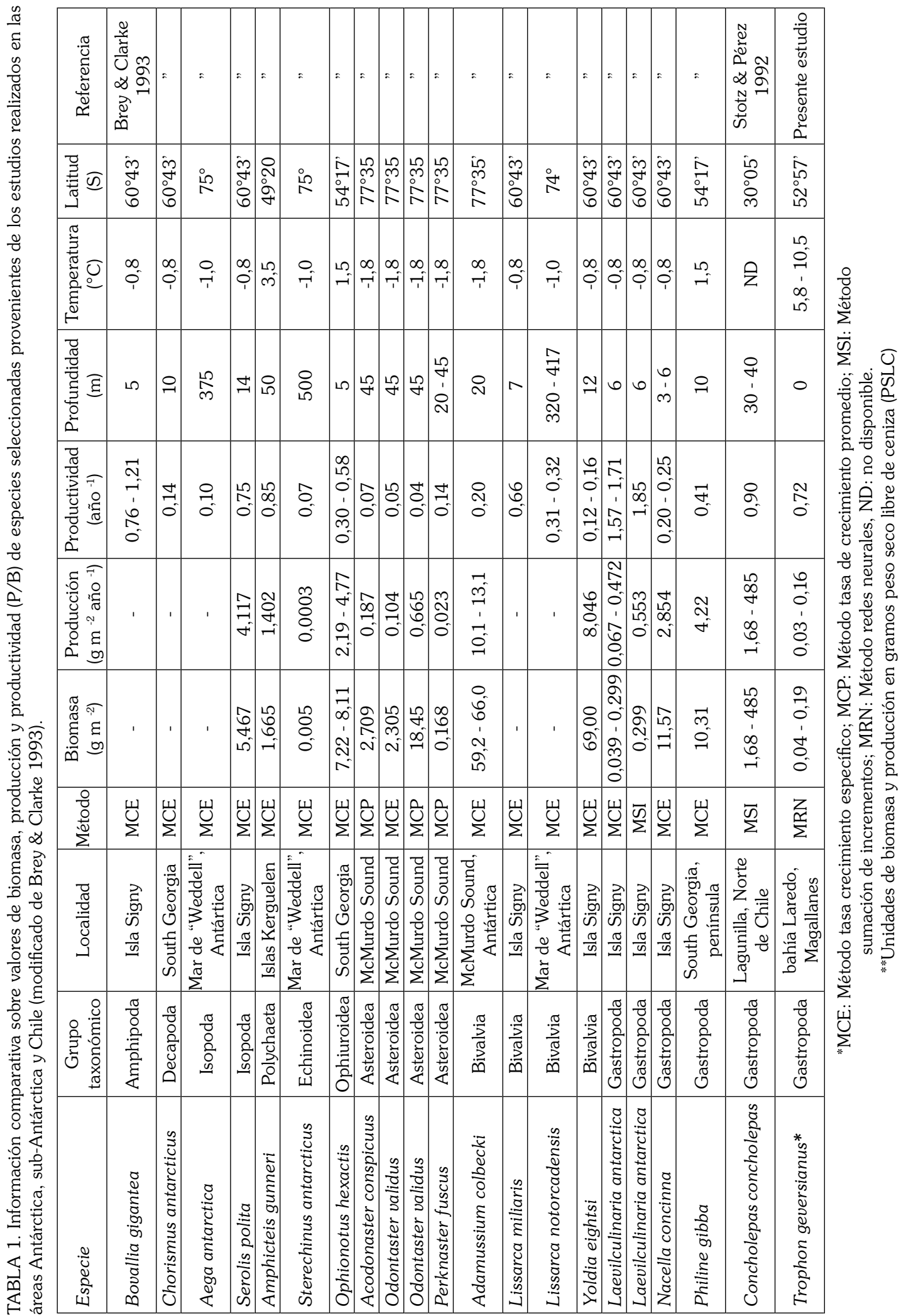


relevantes de la especie provienen de informes técnicos, tesis e información fraccionada en libros. Esto refuerza la necesidad de avanzar en la comprensión de la ecología de $T$. geversianus en el ecosistema marino de la región de Magallanes, a fin de poder contribuir al desarrollo de numerosos aspectos relacionados como lo es el establecimiento de áreas de manejo o en técnicas desarrollo sustentable de los recursos marinos de la región.

\section{AGRADECIMIENTOS}

Los autores agradecen la cooperación del Geógrafo Daniel Antúnez, quién desarrolló parte del SIG utilizado para este estudio. Al alumno de Biología Marina de la Universidad de Magallanes Rodrigo Mancilla por su asistencia en terreno y laboratorio. Igualmente expresamos nuestros agradecimientos al Laboratorio de Hidrobiología del Instituto de la Patagonia, Universidad de Magallanes, por todo el equipo y materiales facilitados para el desarrollo de este estudio. Finalmente a los anónimos revisores que contribuyeron con importantes comentarios al manuscrito.

\section{LITERATURA CITADA}

Acosta, V. \& A. Prieto 2008. Producción secundaria en una población de Perna perna (Bivalvia: Mytilidae) en condiciones de cultivo suspendido. Interciencia 33 (9): 687-692.

Andrade, C. \& C. Ríos 2007. Estudio experimental de los hábitos tróficos de Trophon geversianus (Pallas, 1774) (Gastropoda: Muricidae): Selección y manipulación de presas. Anales Instituto Patagonia (Chile) 35 (1): 45-54.

Asmus, H. \& R. Asmus 1990. Trophic relationships in tidal flats areas: To what extent are tidal flats dependent on imported food?. Netherlands Journal of Sea Research 27: 93-99.

Asmus, H. 1987. Secondary production of an intertidal mussel bed community related to its storage and turnover compartments. Marine Ecology Progress Series 39: 251-266.

Brey, T. 2001. Population dynamics in benthic invertebrates. A virtual handbook. Alfred Wegener Institute for Polar and Marine Research, Germany. Versión 1.2. http://www. thomas-brey.de/science/virtualhandbook/
Brey, T. \& A. Clarke 1993. Population dynamics of marine benthic invertebrates in Antarctic and subantarctic environments: are there unique adaptations? Antarctic Science 5: 253-266.

Brey, T., A. Jarre-Teichmann \& O. Borlich 1996. Artificial neural network versus multiple linear regression: predicting $\mathrm{P} / \mathrm{B}$ ratios from empirical data. Marine Ecology Progress Series 140: 251-256.

Brey, T. \& D. Gerdes 1999. Benthic community productivity in the Magellan region and in the Weddell Sea. Scientia Marina 63 (1): 145-148.

Castellanos, Z. \& N. Landoni 1993. Catálogo descriptivo de la malacofauna marina magallánica 9. Neogastropoda Muricidae y Thaisidae. Comisión de Investigaciones Científicas de la Provincia de Buenos Aires. 25 pp.

Castilla, J. C. \& M. Fernández 1998. Small-Scale benthic fisheries in Chile: On co-management and sustainable use of benthic invertebrates. Ecological Applications 8 (Supplement): S124-S132.

Crisp, D. J. 1984. Energy flow measurements. In: Holme, N.A. \& A.D McIntyre (Eds.), Methods for the Study of Marine Benthos, 2nd ed. Blackwell Scientific Publications, Norfolk. pp. 284-372.

Cusson, M. \& E. Bourget 2005. Global pattern of macroinvertebrate production in marine benthic habitat. Marine Ecology Progress Series 297: 1-14.

Dolbeth, M., A. Lillebo, P. Cardoso, S. Ferreira \& M. Pardal 2005. Annual production of estuarine fauna in different environmental conditions: an evaluation of the estimation methods. Journal of Experimental Marine Biology and Ecology 326: 115-127.

Forcelli, D. 2000. Moluscos magallánicos. Guía de moluscos de Patagonia y sur de Chile. Vásquez Manzini. Buenos Aires. 200 pp.

Gluyas-Millán, M., C. Quiñónez \& J. Talavera 1999. Parámetros poblacionales del caracol Astraea undosa (Word, 1828) en la costa occidental de la península de Baja California. Ciencias Marinas 26 (4): 643-658.

Gordillo, S. \& S. Amuchástegui 1998. Estrategias de depredación del gastrópodo perforador 
Trophon geversianus (Pallas) (Muricoidea: Trophonidae). Malacología 39 (1-2): 83-91. Gray, J. \& M. Elliot 2009. Ecology of marine sediment for science to management. Oxford University Press. $225 \mathrm{pp}$.

Griffin, M. \& G. Pastorino 2005. The genus Trophon Montfort, 1810 (Gastropoda: Muricidae) in the Tertiary of Patagonia. Journal of Paleontology 79: 296-311.

Gutt, J., E. Helsen, W. Arntz \& A. Buschmann 1999. Biodiversity and community structure of the megaepibenthos in the Magellan Region (South America). Scientia Marina 63: 155-170.

Leiva, G. \& J. C. Castilla 2002. A review of the world marine gastropod fishery: Evolution of catches, management and the Chilean experience. Reviews in Fish Biology and Fisheries 11: 283-300.

Linse, K. 1999. Mollusca of the Magellan region. A checklist of the species and their distribution. Scientia Marina 63: 399-407.

Pastorino, G. 2005. A revision of the genus Trophon Montfort, 1810 (Gastropoda: Muricidae) from southern South America. The Nautilus 119: 55-82.

Penchaszadeh, P. 1976. Reproductions of gastropods from southwestern Atlantic. The genus Trophon. Physis Riv Sociedad Argentina de Ciencias Naturales Buenos Aires 90: 69-76.

Prieto, A., L. Ruíz \& H. Hernández 2004. Dinámica energética del crecimiento en una población del mejillón Modiolus squamosus en el golfo de Cariaco, Venezuela. Interciencia 29 (2): 74-79.

Prieto, X. 1988. Geología del Cuaternario en el área de cabo Negro, estrecho de Magallanes, Chile. Anales Instituto Patagonia, Serie Cs. Nat. (Chile) 18: 35-41.

Prince, J., L. Sellers, W. Ford \& S. Talbot 1988. Recruitment, growth, mortality and population structure in a southern Australian population of Haliotis rubra (Mollusca: Gastropoda). Marine Biology 100: 75-82.

Ríos, C. \& D. Gerdes 1997. Ensamble bentónico epifaunístico de un campo intermareal de bloques y cantos en bahía Laredo, estrecho de Magallanes. Anales Instituto Patagonia, Serie Cs. Nat. (Chile) 18: 35-41.
Rodríguez, J., F. Caballero, F. Uribe \& A. Arano 2002. Abundancia y asociaciones de dos gasterópodos (Astraea y Haliotis) comercialmente importantes en isla San Jerónimo, Baja California, México. Ciencias Marinas 28 (1): 49-66.

Santana, M. \& J. Cañete 2001. Antecedentes biológicos para el cultivo del caracol marino Trophon geversianus (Pallas, 1774) (Gastropoda: Muricidae) en Magallanes. Anales Instituto Patagonia, Serie Cs. Nat. (Chile) 29: 105-115.

Santana, M. 1998. Estudios sobre la época de desoves en la naturaleza y desarrollo intracapsular en laboratorio del caracol Trophon geversianus (Pallas, 1774) (Gastropoda: Muricidae). Anales Instituto Patagonia, Serie Cs. Nat. (Chile) 26: 31-40.

Schmitt, R. 1981. Contrasting anti-predator defenses of sympatric marine gastropods (Family Trochidae). Journal of Experimental Marine Biology and Ecology 54: 251-263.

Sprung, M. 1993. Estimating macrobenthic secondary production from body weight and biomass: a field test in a non-boreal intertidal habitat. Marine Ecology Progress Series 100: 103-109.

Stotz, W. \& E. Pérez 1992. Crecimiento y productividad del loco Concholepas concholepas (Bruguiére, 1789) como estimador de la capacidad de carga en áreas de manejo. Investigaciones Pesqueras (Chile) 37: 13-22.

Tumbiolo, M. \& J. Downing 1994. An empirical model for the prediction of secondary production in marine benthic invertebrate populations. Marine Ecology Progress Series 114: 165-174.

Urban, J. H. \& B. Campos 1994. Population dynamics of the bivalves Gari solida, Semele solida and Protothaca thaca from a small bay in Chile at $36^{\circ} \mathrm{S}$. Marine Ecology Progress Series 115: 93-102.

Vermeij, G. 1972. Intraspecific shore-level size gradients in intertidal molluscs. Ecology 53: 693-700.

Zaixso, H. 1973. Observaciones sobre desove y embriología de Trophon geversianus (Pallas, 1774). Neotropica 19 (60): 156-162. 
